\title{
Lived Experiences of Legal Guardians of Adolescents Who Deliberate Self-Harm
}

\author{
Roberto Galea1 $^{*}$, Michael Galea ${ }^{2}$ \\ ${ }^{1}$ Faculty of Medicine and Surgery, University of Malta, Msida, Malta \\ ${ }^{2}$ Department of Mental Health, Faculty of Health Sciences, University of Malta, Msida, Malta \\ Email: *roberto.galea.09@um.edu.mt
}

How to cite this paper: Galea, R. and Galea, M. (2018) Lived Experiences of Legal Guardians of Adolescents Who Deliberate Self-Harm. Open Access Library Journal, 5: e5098.

https://doi.org/10.4236/oalib.1105098

Received: December 5, 2018

Accepted: December 25, 2018

Published: December 28, 2018

Copyright $\odot 2018$ by authors and Open Access Library Inc.

This work is licensed under the Creative Commons Attribution International License (CC BY 4.0).

http://creativecommons.org/licenses/by/4.0/

(c) (i) Open Access

\begin{abstract}
Objectives: To explore and understand the lived experiences of the legal guardians of adolescents who engaged in Deliberate Self-Harm while investigating the need for further education and support. Method: Four participants and one pilot study were purposively sampled from the Child Guidance Unit in Maltaall recruited participants were the biological mothers of self-harming adolescents. Each participant was interviewed twice. Semi-structured interviews were conducted to collect the participants' experiences and subjective perspectives, and they were all digitally recorded. All necessary ethical authorisations were collected. Sample: Participants were parents of adolescents who deliberately self-harmed and were receiving care at the Child Guidance Unit. None of the mothers were living with the adolescents' biological father. Results: The super-ordinate themes identified were: Guardian's depths of despair, Guardians weathering the storm and Hope in the midst of despair. Themes flowed from the discovery of Deliberate Self-Harm and initial reactions to the hopes and skills developed to deal with their respective circumstances. Conclusion: The need to develop better understanding of the traumatisation and difficult scenarios experienced by legal guardians was identified while further support, education and management of Deliberate Self-Harm have been identified. Limited qualitative research exists that has investigated the phenomenon of DSH, especially from the legal guardians' perspective. This research hopefully would assist involved legal guardians how to identify such phenomenon, how to educate themselves about it, and manage it successfully.
\end{abstract}

\section{Subject Areas}

Cognitive Disorders, Psychiatry \& Psychology

\section{Keywords}

Legal Guardians, Lived Experiences, Parents, Hermeneutics, Adolescents, 


\section{Introduction}

Adolescence is characterised by a series of developmental stages during which several social and psychological changes occur. It is in conjunction with these new situations that adolescents tend to start expressing their discomfort through Deliberate Self-Harm (DSH) in order to regulate their emotions [1]. DSH describes an array of deliberate acts resulting in the direct and deliberate harm of one's body, in the absence of any suicidal intentionality [2] [3] [4]. Starker (2006) describes such self-harm ensuing from lack of efficient coping skills. DSH results from maladaptive emotional intelligence [5] and is used to manage emotional and psychological pain [6]. Self-Harming individuals exhibit strong tendencies of repeating DSH, which in turn places them at a higher long-term suicide risk. Familial, genetic and psychosocial components also contribute to DSH [7]. In this study DSH was defined as non-fatal DSH.

A nation-wide study across the United States showed that hospital admissions tripled from 1990 to 2000 while the prevalence rate for DSH in adolescents increased from 3\% in 1993 to $15 \%$ in 2002 [8]. 59\% of those admitted into hospitals report pervious episodes of DSH, while $30 \%$ were between 15 to 24 years of age. Eternal causes of death are the third leading cause of death in the European Union (7\% of all deaths). Suicide and motor vehicle accidents were the leading causes of external deaths in 2014; however in 2015 suicide became the leading external cause of death which continued to represent a major public health problem with an average of 10.4/100,000 inhabitants. Overall, external causes of death decreased from 82 to 50/100,000 inhabitants between 2000 and 2015 [9]. Death by suicide in Malta shifted from 4.49 (2011) to 5.05 (2013) to 7.64 (2015) per 100,000 inhabitants. In 2015, Lithuania (30.28), Slovenia (20.71) and Latvia (19.34) reported the highest incidence of suicide in the European Union, while Turkey (2.18), Liechtenstein (2.48) and Cyprus (4.45) had the lowest rates. Such low incidences may reflect missing data, lack of reporting or cultural protective factors [10].

According to the Directorate for Health Information and Research, the number of reported suicides in Malta progressed from 22 (2013) to 28 (2014) and 32 (2015) with in the age group 15 - 44 years only. A difficulty to ascertain whether a death was intentional or accidental was reported. Suicides are still under-reported and not always written on the death certificate. Those reported are cross-checked by the National Mortality Register with the Police Statistics Office [11]. Unemployment was on a continuous decline from January 2015 and reached a maximum low in September 2018 [12], however in the same period, death by suicide was on the incline. To date, no local data has been found reporting non-suicidal intentional DSH. 
In the field of DSH, lived experiences are not yet fully explored by the literature [4]. A literature review yielded 99 studies dealing with lived experiences of legal guardians of adolescents who deliberately self-harm; identifying a niche in the literature necessitating further research [13] [14] [15]. DSH is extremely debilitating for both the individual and their relatives who face numerous difficulties to assist the self-harming individual while function within society [4]. Understanding such a phenomenon assists in the identification of areas in which legal guardians require further support such as education, management and coping skills. To date, locally no such research has been conducted.

\section{Methodology}

\subsection{Aims and Objectives}

The aims of this study were to develop an understanding of the lived experiences of legal guardians whose adolescents deliberately self-harm. This understanding would avail legal guardians for a better comprehension, education and for learning better skills how to manage such a phenomenon. A qualitative approach was utilised since it assists the researcher to grasp each participants' unique circumstances and subjective realities [16]; thus assisting the researcher to develop an understanding of human experience and its context which influences one's perception, behaviour and motivation [17]. Interpretative Phenomenological Analysis as interpreted by Smith, Flowers and Larkin (2009) was used due to the subjective nature of the data collected.

\subsection{Data Collection}

\subsubsection{Sample and Pilot Study}

A pilot study was done to refine the interview protocol. Later on, a purposive homogeneous sample of four participants was recruited. In order to be recruited, legal guardians had to be of eighteen years of age or older and legally responsible for adolescents attending the Child Guidance Unit (CGU) who engaged in DSH in the past year. Additionally, potential participants were approached by an intermediary from the CGU.

\subsubsection{Semi-Structured Interviews}

Audio-recorded, in-depth semi-structured interviews were conducted. Semistructured interviews provided an increasingly fluid interview, enabling the researcher to engage with the interviewee in a phenomenological conversation and focus the interview on issues the participants perceived as important [18]. During the interview participants became collaborators and formed a conversational relationship, involving the interviewee in their experience of the phenomenon while keeping the personal element in the conversation [17]. Audio-recordings allowed the researcher to repeatedly go through each interview until each participants' experiences was fully understood and interpreted in its context.

During the interview attention was given to neutral, unbiased statements, refraining from influencing the participants to answer in a manner desired by the 
researcher. A second interview was conducted four weeks after the first interview, during which the data collected was analysed and new questions for each participant developed to probe further into each case.

\subsection{Trustworthiness, Credibility and Reflexivity}

Trustworthiness and credibility were achieved by transcribing all audio-recordings and repeatedly read the transcripts. A second interview probed further into the experiences, verified previously established knowledge and increased the credibility and faithfulness to the participants' accounts [19]. Honesty was an important value to ensure respect towards both the individuals and their accounts while ensuring the genuineness of the inferred interpretations. Throughout the study the researcher abided to the four principles put forward by Yardley (2000), these being;

1) Sensitivity to context

2) Commitment and rigour

3) Transparency and coherence

4) Impact and importance

These are considered to improve the quality of qualitative research and ensure a neutral and consistent interpretation of data while respecting the participants and their experiences. A reflective diary was kept throughout the study to keep track of all the decisions taken and amendments made, particularly the feelings experienced during the interviews.

\subsection{Ethical Considerations}

All ethical approvals were strictly adhered to by the researcher. To help the participants feel safer and not pressured to participate, participants were approached by an intermediary at the CGU. Participants were given an information letter and a consent form and were informed that they could withdraw at any point without providing any rationales with no repercussions for themselves or their adolescent. Strict confidentiality was maintained throughout. To ascertain confidentiality, pseudo-names were given to the participants. The data collected was stored in a password protected file and destroyed as soon as the study was completed. Psychological services were on offer, if the need arose.

\section{Results (Table 1 \& Table 2)}

Table 1-Participants' Demographics; The sample consisted of four biological mothers (plus one pilot study) of adolescents between the age of 13 to 15 years of age with a history of deliberate self-harm and currently being followed-up by a child and adolescent psychiatrist at the Child Guidance Unit. At the time of the interview, none of the mothers and adolescents lived with the biological fathers while all adolescents had recently experienced traumatic events in their lives.

The following are the themes highlighted by the participants during the study, presented according to their respective super-ordinate themes. Table 2 outlines 
Table 1. Participants' demographics.

\begin{tabular}{|c|c|c|c|c|c|c|c|}
\hline Participant & Adol. Gender & Type of DSH & Stopped DSH & $\begin{array}{c}\text { Suicidal } \\
\text { Attempt/s }\end{array}$ & Trauma/s & Parents & Participant \\
\hline Pilot & Female & Self-Mutilation & Yes & Nil. & Lifestyle alteration & $\begin{array}{c}\text { Biological - } \\
\text { Married }\end{array}$ & Mother \\
\hline$\# 1$ & Female & Self-Mutilation & Yes & 1 & Bullying & $\begin{array}{l}\text { Biological - } \\
\text { Separated }\end{array}$ & Mother \\
\hline$\# 4$ & Female & Self-Mutilation & No & Nil. & Relationship with Father & $\begin{array}{l}\text { Biological - } \\
\text { Separated }\end{array}$ & Mother \\
\hline$\# 7$ & Male & Nil. & Yes & 2 & Relationship with Father & $\begin{array}{l}\text { Biological - } \\
\text { Separated }\end{array}$ & Mother \\
\hline$\# 8$ & Female & $\begin{array}{l}\text { Pressing asthma inhaler } \\
\text { against stomach }\end{array}$ & Yes & Nil. & Witnessed father's affair & $\begin{array}{l}\text { Biological - } \\
\text { Separated }\end{array}$ & Mother \\
\hline
\end{tabular}

Table 2. Identified themes.

\begin{tabular}{cc}
\hline Super-Ordinate Themes & Themes \\
Guardians' depths of despair & Adolescents \\
Trauma & Coping, Anxiety \& Attention seeking \\
Guardians weathering the storm & Endurance \\
& Socioeconomic deprivation \\
& Familial relationships \\
Hope in the midst of despair & Spirituality \\
\end{tabular}

the super-ordinate themes identified from the participants' perceived lived experiences following Interpretative Phenomenological Analysis.

\subsection{Guardians' Depths of Despair}

\subsubsection{Adolescents' Traits}

Participants reported their adolescents' atypical traits such as; low self-esteem, impulsivity, negative attitudes, lack of consideration and disruptive relationships as troublesome and difficult to cope with. Despite their dedication and effort to help their adolescents improve and eliminate DSH, the mothers disclosed the daily afflictions they encountered. Dealing with challenging and manipulative adolescents, while trying to communicate and maintain a relationship with them was strenuous.

The adolescents' disruptive traits and emotional turmoil made it difficult for the participants to engage with and understand their perspectives. Quarrels resulted, whereby the adolescents blamed responsibility to their mothers, which made them feel helpless. Mothers described developing management and interpersonal skills through experience and never being professionally instructed; as Lisa distressingly recounted: 
"Mmm, he was doing everything to attract attention. He started, mmm, creating a lot of problems, even with school, he didn't want to attend. This means, these last three years the situation got worse!... If he start putting ideas into his head, he sees how to actualize them... For example, about school, he had been grumbling about leaving school for some time. He succeeded because eventually he stopped going to school. Mmm, he tells me up to this day when he has one of those days when he has a bad mood, that it is my fault that he ended up like this..."

\subsubsection{Trauma}

Experiences identified as triggering DSH were; significant lifestyle changes, bullying, the separation and loss of significant others, traumatic events or sudden environmental alterations. These circumstances were already challenging for the mothers themselves, the manifestation of DSH made things worse; increasing turmoil within their relationships and socio-economic concerns such as financial hardship and expenses. Mothers described noticing both subtle and abrupt behavioural changes and subsequently identifying the cause of such changes. Mothers tended to question themselves, what was wrong with their child, the decisions they made and their parenting skills. The discovery of DSH was traumatising for the mothers who had to deal with the causing factor, their traumatised adolescents and themselves which brought successive changes to their lives and relationships. Donna explained that she had different children from different partners, a situation which led to an unstable lifestyle and upbringing of her children. Consequently, Donna feels responsible for her child's DSH, arousing guilt feelings:

"Yes, I feel I did some wrong doing. For example, I was fourteen years old when I had my first baby, afterwards I broke up from my partner. Do you understand? Then I got married and while pregnant with the second child, I broke up again from my second partner..."

Despite these problematic circumstances, Donna's children felt lucky to have her in their lives and further indicated her children's ambition to become like her in the future. These statements further confused the mother since she did not understand how she could be their role-model. When Donna's partner abruptly stopped visiting his child due to a relationship with another woman, this had devastating consequences. Allegedly, the adolescent experienced "homosexuality" as a defence mechanism to avoid being hurt by other men. However, it was discrimination by others, not "homosexuality" which preoccupied Donna.

Donna feels it is her fault that the family was not united and felt responsible for the incidents her children experienced. As a result of the responsibility and commitment towards her adolescent Donna changed her working hours. Hence, when her daughter returned from school she had somebody to speak, associate with and externalise any distressing emotions. Donna also stated that she tried not to become angry when her child self-harmed but in reality she felt defenceless and incapable of dealing with such situations. 
It was identified that adolescents do not recognise the need for psychiatric intervention and argue with both their parents and professionals regarding the usefulness of such consultations. Participants understood that attending psychology sessions against their children's will, would not be beneficial. The mothers described that with regards to psychiatric reviews their adolescents kept their appointments, even against their wishes. Prior the second interview Michelle's daughter was started on methylphenidate which increased her attention and concentration noticeably. Resultantly her daughter recognised the positive effect this had on her lifestyle and became increasingly compliant to treatment.

\subsubsection{Coping, Anxiety \& Attention Seeking}

Only Lisa associated her adolescent's DSH to an attention seeking mechanism. The other participants reported that DSH was a means of coping, achieving a sense of relief from accumulated anxiety. Although not rationally understanding why adolescents self-harmed, mothers had to accept it. This distressed the mothers since they felt defenceless in front of their adolescents and unable to help them. Social isolation was experienced due to the fear of their adolescents' reactions and uncertainty how to manage them in public. Mothers were afraid of being seen as incapable to parent appropriately by outsiders.

Disruptive relationships denoted the lives of these adolescents. Some participants described subtle inclinations while others reported full blown confrontational arguments. The main rationales identified resulted either due to trust issues, coping mechanisms or personality traits. Lisa described that after a difficult day her son is very confrontational, insulting and inquisitive. During such quarrels her son tells her that all that he had been through resulted from the bad decisions she made, arousing guilt feelings and her questioning her parenting abilities. As Lisa disclosed:

"... when he is having a bad day he tells me things he starts spurting things out, and he becomes aggressive... he says a lot of things and they hurt. And he utters them so that he can be offensive. He tells me that it is my fault..."

Michelle described her adolescent as perseverant, no particular concerns in her relationship with her daughter or any other disruptive traits. However, she remarked that her adolescent did not stick to the same social group for long periods of time, either because she became fed up or due to quarrels. Several participants admitted that their marital situation and family dynamics had been significant influencing factors in the manifestation and progression of DSH.

\subsection{Guardians Weathering the Storm}

\subsubsection{Endurance}

The toughest moment participants endured was the discovery of DSH, while the discovery of attempted suicide was far more devastating for the child, the discoverers and the whole family unit. Individuals who discovered the incident vividly reported the instance and relived the emotional turmoil experienced through 
narration. Although in all cases these episodes were not recent, clearly these left an impact on the participants' lives and perception of their families and surroundings. Lisa experienced two attempted suicides by her son. The first episode took place at home while the second one two weeks later at school. Lisa was informed of the happenings through a phone call and rushed to her child at hospital. The mother also recalled another incident when she grabbed her son from a window sill where he was allegedly attempting suicide. Even though Abbi did not discover the incident, this was enough traumatic to her that just by recounting the event, it elicited an emotional response. Abbi recognised the fact that her partner and daughter had gone through a much worse trauma than herself. Another sentiment these mothers experienced when discovering DSH was that they questioned themselves, their upbringing and behaviours, arousing feelings of helplessness, defenceless and failure.

Participants admitted that it had been a though period to get over, manage and cope. Mothers were still accountable for a number of duties and held social, financial and familial responsibilities while supporting their adolescents. In fact, during the interviews several mothers became distressed, upset and emotional while narrating their experiences; Michelle experienced mental exhaustion as a result of continually dealing with strenuous issues, as she disclosed:

"But you suffer a lot, I felt overwhelmed because $1 \mathrm{~m}$ telling you I was mentally destroyed, because physically and mentally if your mind doesn't function well you are going to be really exhausted!"

Abbi exhibited a remarkable need to share her experiences and willingly expressed her genuine interest in this process. Abbi denoted the importance given to her daughter's achievements as a family initiative to help develop the adolescent's skills and proving her worth. As a result of the bullying her daughter suffered at school, Abbi was looking forward for her child finishing secondary school and stop being bullied.

"She won! As she went back on stage she asked me to join her! I told her don't let anyone underestimate your worth! After all that bullying, she was really satisfied. We won because she won herself back! I think that was the best moment... and we both shed tears of joy on stage!"

These mothers tried hard to adapt to their new circumstances. In the absence of proper understanding of DSH, participants tried to inform themselves and adequately behave with their adolescents, trying to accept their adolescents as much as they could. Lack of discipline and control over these adolescents was also observed. This was described by a number of participants to result from fearing unforeseen responses. Mothers engaged in such behaviour due to their inappropriate and undeveloped skills in dealing with these situations. Abbi tried to manage her daughter as best she knew and taught her that life was a beautiful and meaningful experience. However, lack of commitment from these adolescents was disregarded due to the fear of DSH. Participants disclosed that they felt helpless in managing their adolescents and were aware of the absence of discipline. 


\subsubsection{Socioeconomic Deprivation}

Financial burdens and social isolation resulted due to stigma, private sessions and medication. Socio-economic issues were magnified since mothers reduced their working hours to care for their adolescents. Lisa felt anguished and frustrated due to the general public's lack of understanding, this furthered feelings of isolation:

"The people do not understand and unintentionally they put the blame why this happened on something or someone."

Stigma may be exhibited subtlety, in fact Lisa described stigma as a lack of understanding of one's situation. Lisa felt discriminated as a single parent and for her unsuccessful relationship with her spouse. Lisa further elaborated that constantly repeating her story and having to justify every decision to validate herself as distressing. No other participant described experiencing stigma; however, stigma was perceived as negatively affecting both the services users and their care-givers.

Michelle described that it was difficult to meet daily expenses while supporting her adolescent through these circumstances. The other two participants did not verbally describe financial difficulties; nonetheless made references to financial struggles especially with private health-care and medications.

\subsubsection{Familial Relationships}

Participants reported that following the initial shock, relationships within the family were not affected. Participants recalled persistently looking for potentially dangerous objects amongst their adolescent's belongings and searching social media for references to DSH, resulting in hyper-vigilance.

Hyper-vigilance was misinterpreted for care. Although some participants recognised hyper-vigilance as inadequate and subsequently tackled it, fragments of caution and suspicion persisted. Despite the mothers stopped invading their adolescents' privacy, persistent preoccupations and trust issues ensued. As a result of DSH, mothers invested more effort in their relationship with their self-harming adolescent with regards to time, availability, patience and consideration, thus relationships "benefited" from DSH. Abbi further argued:

"Then we decided to risk it. We thought it was not realistic at all. We had all forks and knives removed. How was she going to eat if we were not at home? How is this child meant to feel any better in these conditions?!"

Mothers described being readily available for their children by changing their working hours or stop working. Time management was an incessant difficulty due to the participants' demands with respect to employment, family and partners but not themselves. Despite all their duties and time limitations, participants managed their lives successfully and were astonished at the duties they managed. Lisa stated that if she did not manage, no one will-portraying her commitment to her son and their lives together. Participants seldom mentioned any personal issues other than their adolescents' health and reported that their issues were only familial issues. 
Religious beliefs were repeatedly noted by Abbi as important, denoting her locus of control. DSH was normalised amongst the participants facilitating coping, establishing an adequate level of understanding and assist burden sharing. Lisa realised how subtle the change required to alter one's mental status and instigate DSH was.

\section{Discussion}

This study provides an in-depth exploration of the lived experiences of legal guardians of adolescents who deliberately self-harm. Following the discovery of DSH, participants experienced the "depths of despair" as they were; in shock, had never considered they could experience such a situation and had no measures to cope with such circumstance. However, by time, through their own knowledge and literature available to them, participants tried to make sense out of their situation and with the help of professionals started tackling their issues; thus they felt as "weathering the storm". The recruited participants described that thanks to the incessant support received from both their families and professionals, participants started to have "hope in the midst of despair", could further develop their relationships and were in a better position to assist their adolescents.

\subsection{Guardians' Depths of Despair}

Care-givers of self-harming adolescents meet the criteria for minor psychological distress and rate the severity of their children's circumstances as abnormally high [20]. Their disruptive relationship arouses feelings of helplessness, guilt and defencelessness. The responsibilities and workload of these mothers prior the discovery of DSH was already considerably high-increased stress hindered the relationships with their adolescents. The most disrupting factor accompanying adolescent DSH was its discovery, and participants were at a loss how to react. Their poor relationships made DSH harder to manage and accept. Participants noticed both subtle and abrupt changes in their adolescents' behaviour prior discovering DSH and acknowledged that changes had been going on for a long period of time, followed by quarrelling which led mothers to question their parenting skills. Parents underestimated the significance of warning signs and tried to observe their adolescent more attentively hoping they misinterpreted these signs [21]. This delayed the sequence for help.

Mothers described having good relationships with their adolescents. However, reported placing much more effort into their relationships after discovering DSH. Although parents were committed to their adolescents, they were more focused on other issues prior the discovery of DSH. Appleyard and Osofsky (2003) reported that in these cases parents may experience symptoms related to Post-Traumatic Stress Disorder (PTSD). PTSD impairs their abilities to function and fulfill their roles resulting in persistently negatively stimulating individuals surrounding them [22] and affecting their adolescents' biopsychosocial spheres 
who are instigated to engage in dysfunctional coping mechanisms like DSH [22] [23].

Parents enduring similar situations are sensitive to the behaviour surrounding them and hugely affected by their previous experiences [24]. Adolescent DSH has been reported to be traumatic and to trigger feelings of helplessness, anger and failure [20], these further impair the parents' abilities to function, affecting their biopsychosocial spheres, reinforcing DSH in their adolescents-creating a vicious circle. Figure 1 has been developed from the legal guardians' lived experiences and demonstrates the correlation matrix of key variables described above. The fact self-harming adolescents lack the initiative to seek support may result from the aetiology of DSH. Through DSH adolescents externalise emotional stress and release accumulated psychological pain due to lack of coping skills and under-developed emotional intelligence-the elimination of DSH would thus result in the accumulation of such stress. To eliminate DSH adolescents should be taught other effective coping skills while receiving therapy to adequately manage their psychological pain [20].

Participants tried to identify triggers of DSH in order to understand their adolescents' situation. Despite all their efforts, participants did not fully understand this behaviour. Mothers had to live with this distressing issue which made them feel anxious and incompetent. The participants' marital status created additional responsibilities resulting in less time spent with their adolescents. Such responsibilities were met to the detriment of their family and relationships. These undeveloped management skills were compensated by drastic reductions

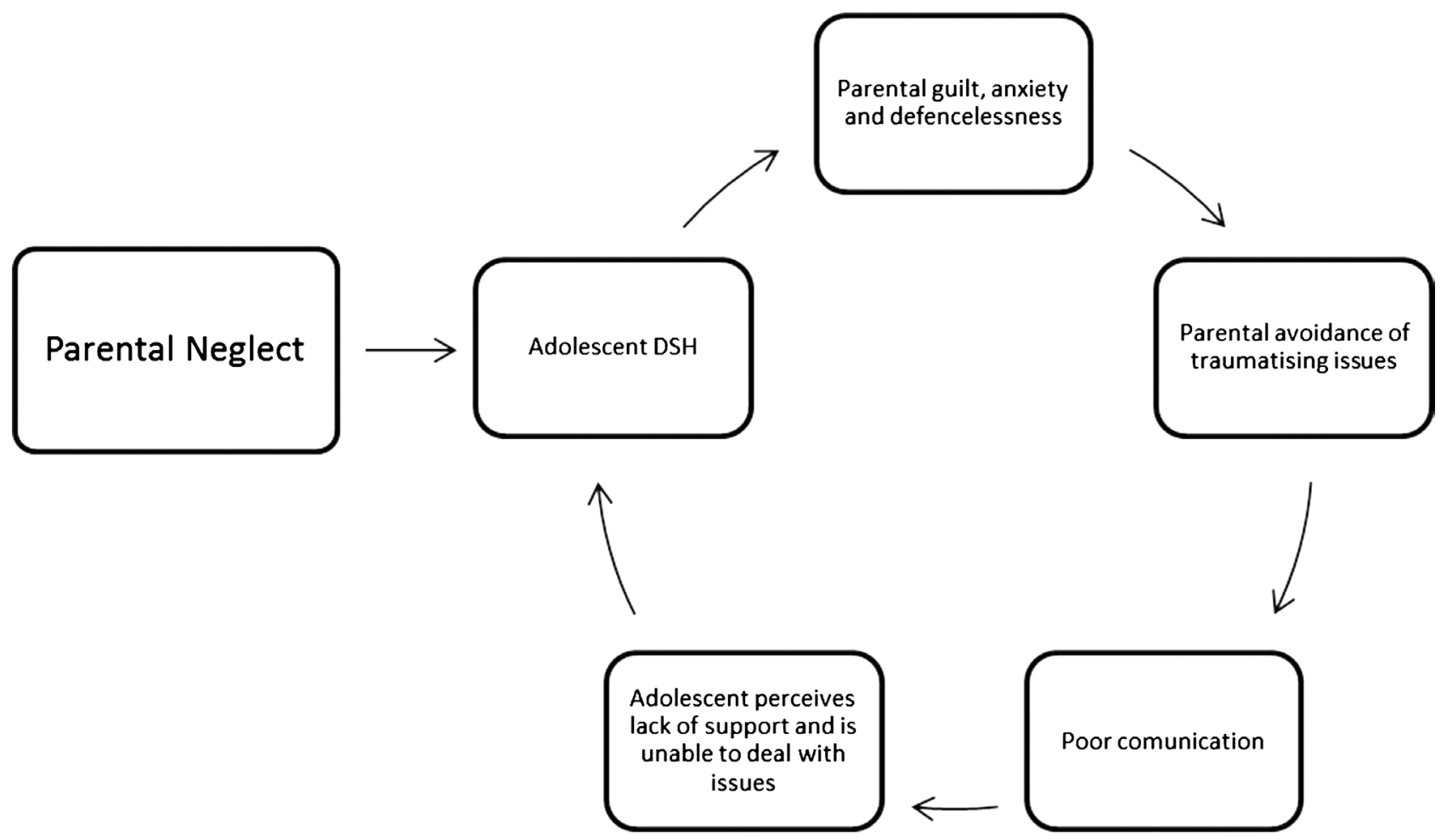

Figure 1. Proposed cycle of deliberate self-harm. 
in the mothers' social life resulting in social isolation. Once parents felt unable to manage their adolescents, parents refrained from social gatherings fearing to confront their parenting skills and being criticised as incapable parents by significant others. Morgan, et al. (2013) stated that parents expressed concerns regarding their parenting skills, competence and abilities to cope.

To stop feeling vulnerable, parents incapable of tackling their adolescents' distress, avoid facing their child's aggression, anxiety and confrontations [22], resulting in lack of communication and increased discordance [20]. The mothers' perception of their families and surroundings was altered by the discovery of DSH [24].

\subsection{Guardians' Weathering the Storm}

Parental difficulties stemming from adolescent mental health have been identified as the caregiver's burden [20]. Increased stress levels routinely underpinned the lifestyle of these mothers. Mothers expressed feelings of burn-out, resulting in taking life one day at a time. Parental health has been found to be lower when the child's difficulties were greater [20], hindering the relationship with their adolescents and indicating that although parents do not seek psychiatric support they still needed it. Parents' were holistically affected by the devastating experiences of adolescent DSH [24]. Participants questioned themselves, their upbringing and decisions to identify what triggered DSH. Although visibly affected by this trauma, participants admitted that they met their parental responsibilities at the detriment of their health.

Social deprivation resulted in the participants' fear of being judged as having failed as parents. Coping with the outside world was not a welcome question during the interviews. Poor family functioning and limited social support correlated to adolescent depression [20]. It is unclear whether participants managed to find time for their adolescents to the detriment of other relationships or working hours prior the discovery of DSH. Mothers either acted out of guilt feelings or they acted on the immediate need to actively tackle their adolescents' situation. Mothers dedicated more time to their adolescents at the price of their own. When mothers neglected their needs, their perceived stress increased [24].

Adolescent DSH is affected by the parents' behaviour [25]. Participants worked at restabilising their family dynamics, normalising the issue and avoid further stress. Improvement of familial relationships reflects the understanding of the adolescents' needs. The mothers described that their relationships 'benefited' from this experience because it helped unify the family [24]. According to Morgan, et al. (2013) DSH produces disruptive concerns and stimulates the development of trust issues between the parents and their adolescents; however, participants in this study did not express any of these issues. This discrepancy may result from the fact that DSH was contained and participants perceived familial relationships as a protective factor thus reducing the effect of this debilitating factor. A complex relationship exists between family dynamics and ado- 
lescent DSH, each determining and protecting one another [20]. Poor communication instils a sensation of social isolation in adolescents, perceiving their issues to be above their ability to solve and engage in DSH [20], emphasising that good family relationships are protective against DSH. Parents should not express shock when discovering DSH as this will negatively reinforce DSH by making the adolescents feel misunderstood. Normalisation, acceptance and support are of outmost importance. Professional help is to be sought to teach effective coping skills, emotional regulation and communication skills. Additionally, support should be provided consistently even after DSH stops to avoid that adolescents resume to DSH as an attention seeking mechanism.

The inability to maintain discipline did not necessarily result only from the inability to understand and manage their adolescents. Hypersensitivity could also have been exhibited to avoid triggering other episodes of DSH. Parents modify their parenting styles to satisfy their adolescents' needs without confronting them [24], inevitably resulting in control issues and hyper-vigilance. Religious beliefs were expressed as being of outmost importance by one participant, delineating an external locus of control.

\subsection{Hope in the Midst of Despair}

Despite effectively coping with these situations, feelings of defencelessness, helplessness and guilt persisted-sentiments stemming from lack of education and support. Education would provide the necessary understanding and management skills of DSH while professional support would tackle symptoms of PTSD. Children of parents exhibiting these emotions suffer from trust and emotional issues, effecting their emotional development too [22]. Although mothers deepened their insight, their management skills did not improve. Parents were aware of the persistent lack of discipline they exercised, however did not know how to approach their adolescents and feared triggering other DSH episodes. Group therapy, support groups and professional guidance would help parents develop their parenting skills and assertiveness [24].

\section{Strengths and Limitations}

This study recruited solely four participants, facilitating the management of data collection and interpretation, however recruiting more participants could have possibly shed light on other subjectively experienced aspects. The homogeneity of the sample provided an in-depth understanding of the recruited group; however, results are exclusive to such population. A better understanding could be developed if a diversity of homogenous samples were investigated. Generalisability was not the aim of the study; however the knowledge developed is transferable to other similar populations.

Although interviews were carried out in Maltese, these were than translated to English by an independent translator. Translation might have led to the essence of a subjective meaning to be lost; thus in situations where this was foreseen, the 
original text in Maltese was retained. The data portrays importantly perceived issues by the participants rather than the researcher. The rigour, quality and genuineness of the findings reported depended on both the feedback given by the participants and the ability of the researcher to extrapolate and interpret the collected data.

The researcher is a novice researcher, this may have reflected upon the interpretation and extrapolation of data. Finally, a very limited amount of research investigating the lived experiences of this specific niche was identified, hence limited studies back up this research; emphasising the need for further research to fully understand this phenomenon which vastly effects adolescents who will form tomorrow's society.

\section{Conclusion}

This study looked into the lived experiences of biological mothers of deliberately self-harming adolescents. For a mother, living, caring and understanding a self-harming adolescent has been described to be: initially traumatic, resultantly life changing and continuously challenging to adapt to the adolescent's behaviour, combat stigma and endure social judgement. Discovery and acceptance of the situation was described as the most difficult part of the whole experience, however lifelong adjustment brought about positive change. These mothers would immeasurably benefit from adequately provided education with regards to both understanding the psychiatric component of the issue and the management of their respective situations. The support of community services and group therapy for both the mothers and their adolescents would further consolidate the therapy received.

\section{Acknowledgements}

We thank all the participants who accepted to share their experience with us, the staff who assisted in the recruitment of participants and development of the study and the research supervisor for his dedication towards students and research.

\section{Funding}

This research has received no specific grant from any funding agency in the public, commercial or non-profit sectors.

\section{Conflicts of Interest}

The authors report no conflicts of interest.

\section{References}

[1] Cerutti, R., Manca, M., Presaghi, F. and Gratz, K.L. (2011) Prevelance and Clinical Correlates of Deliberate Self-Harm among a Community Sample of Italian Adolescents. Journal of Adolescence, 34, 337-347. 
https://doi.org/10.1016/j.adolescence.2010.04.004

[2] Krikcaldy, B.D., Eysneck, M.W. and Siefen, G.R. (2004) Psychological and Social Predictors of Suicidal Ideation among Young Adolescents. School Psychology International, 25, 300-316. https://doi.org/10.1177/0143034304046903

[3] Nock, M.K., Teper, R. and Hollander, M. (2007) Psychological Treatment of Self-Injury among Adolescents. Journal of Clinical Psychology, 63, 1081-1089. https://doi.org/10.1002/jclp.20415

[4] Trepal, H.C., Wester, K.L. and MacDonald, C.A. (2006) Self-Injury and Postvention: Responding to the Family in Crisis. The Family Journal, 14, 342-348. https://doi.org/10.1177/1066480706291128

[5] Mikolajczak, M., Petrides, K.V. and Hurry, J. (2009) Adolescents Choosing Self-Harm as an Emotional Regulation Strategy: The Protective Role of Trait Emotional Intelligence. British Journal of Clinical Psychology, 48, 181-193. https://doi.org/10.1348/014466508X386027

[6] Starker, G. (2006) Signing with a Scar: Understanding Self-Harm. Psychoanalytic Dialogues, 16, 93-112.

[7] Soomro, G.M. (2006) Deliberate Self-Harm (and Attempted Suicide). Clinical Evidence, 12, 1-29.

[8] Jacobson, C.M., Muehlenkamp, J.J., Miller, A.L. and Turner, J.B. (2008) Psychiatric Impairment among Adolescents Engaging in Different Types of Deliberate Self-Harm. Journal of Clinical Child and Adolescent Psychology, 37, 363-375. https://doi.org/10.1080/15374410801955771

[9] World Health Organization-Europe (2018) European Health Report 2018.

[10] Eurostat (2016) Causes of Death Statistics: 2015.

[11] Directorate for Health Information and Research (2015) Annual Mortality Report: 2013.

[12] National Statistical Office (2018) Registered Unemployed: September 2018.

[13] Chesley, K. and Loring-McNulty, N.E. (2003) Process of Suicide: Perspective of the Suicide Attempter. Journal of the American Psychiatric Nurses Accosication, 9, 41-45. https://doi.org/10.1016/S1078-3903(03)00051-X

[14] Fortune, S., Sinclair, J. and Hawton, K. (2008) Adolecents' Views on Preventing Self-Harm. Social Psychiatry and Psychiatric Epidemiology, 43, 96-104. https://doi.org/10.1007/s00127-007-0273-1

[15] Wright, J., Briggs, S. and Behringer, J. (2005) Attachment and the Body in Suicidal Adolesents: A Pilot Study. Clinical Child Psychology and Psychiatry, 10, 477-491. https://doi.org/10.1177/1359104505056310

[16] Pratt, M. (2012) The Utility of Human Sciences in Nursing Inquiry. Nurse Researcher, 19, 12-15. https://doi.org/10.7748/nr2012.04.19.3.12.c9053

[17] Nicholls, D. (2009) Qualitative Research: Part One-Philosophies. International Journal of Therapy and Rehabilitation, 16, 526-533. https://doi.org/10.12968/ijtr.2009.16.10.44562

[18] Smith, J.A., Flowers, P. and Larkin, M. (2009) Interpretative Phenomenological Analysis: Theory, Method and Research. SAGE Publications, Thousand Oaks.

[19] Burhans, L.M. and Alligood, M.R. (2010) Quality Nursing Care in the Words of Nurses. Journal of Advanced Nursing, 66, 1689-1697. https://doi.org/10.1111/j.1365-2648.2010.05344.x

[20] Morgan, S., Rickar, E., Noone, M., Boylan, C., Carthy, A., Crowley, S., et al. (2013) 
Parents of Young People with Self-Harm or Suicidal Behaviour Who Seek Help-A Psychosocial Profile. Child and Adolescent Psychiatry and Mental Health, 7, 1-10. https://doi.org/10.1186/1753-2000-7-13

[21] Thomson, S., Marriott, M., Telford, K., Law, H., McLaughlin, J. and Sayal, K. (2014) Adolescents with a Diagnosis of Anorexia Nervosa: Parents' Experience of Recognition and Deciding to Seek Help. Journal of Clinical Child Psychology and Psychiatry, 19, 43-57. https://doi.org/10.1177/1359104512465741

[22] Appleyard, K. and Osofsky, J.D. (2003) Parenting after Trauma: Supporting Parents and Caregivers in the Treatment of Children Impacted by Violence. Infant Mental Health Journal, 24, 111-125. https://doi.org/10.1002/imhj.10050

[23] Neacsiu, A.D., Rizvi, S.L., Vitaliano, P.P., Lynch, T.R. and Linehan, M.M. (2010) The Dialectial Behaviour Therapy Ways of Coping Checklist: Development and Psychometric Properties. Journal of Clinical Psychology, 66, 563-582.

[24] Oldershaw, A., Richards, C., Simic, M. and Schmidt, U. (2008) Parents' Perspectives on Adolescent Self-Harm: Qualitative Study. The British Journal of Psychiatry, 193, 140-144. https://doi.org/10.1192/bjp.bp.107.045930

[25] Sim, L., Adrian, M., Zeman, J., Cassano, M. and Friedrich, W.N. (2009) Adolescent Deliberate Self-Harm: Linkages to Emotion Regulation and Family Emotional Climate. Journal of Research on Adolescence, 19, 75-91.

https://doi.org/10.1111/j.1532-7795.2009.00582.x 